\title{
Temporary employment and tooth loss: a cross-sectional study from the J-SHINE study

\author{
Yukihiro Sato ${ }^{1}$, Toru Tsuboya ${ }^{1 *}$, Richard G. Watt ${ }^{2}$, Jun Aida ${ }^{1}$ and Ken Osaka ${ }^{1}$
}

\begin{abstract}
Background: Temporary employment leads to psychological distress and higher mortality, but data on its associations with oral health is limited. We examined whether having the experience of temporary employment was associated with tooth loss among working adults in Japan.

Methods: We conducted a cross-sectional study from the 2010-2011 Japanese Study on Stratification, Health, Income, and Neighborhood study that analyzed 2652 participants aged 25-50 years ( men $=1394$; women $=1258$ ). Independent variable was changes in employment status (continuous regular employment and the experience of temporary employment). Dependent variable was self-reported tooth loss (none, 1 tooth, 2 teeth, 3 teeth, 4 teeth, and more than 4 teeth). Covariates were sex, age, years of education, self-rated household economic status in early life at 5 years old, marital status, number of family members in the household, history of diabetes, and body mass index. We conducted a negative binomial regression analysis to estimate prevalence rate ratios (PRRs) and 95\% confidence intervals $(95 \% \mathrm{Cls})$ for tooth loss. We also confirmed the interaction term between changes in employment status and sex.

Results: The median age of the participants was 37 years. The percentages of men and women who experienced temporary employment were $14.5 \%$ and $61.3 \%$, respectively. Compared with continuous regular employment, the experience of temporary employment was significantly associated with tooth loss in both sexes after adjusting for the covariates (men: $\mathrm{PRR}=1.50[95 \% \mathrm{Cl}=1.13,2.00]$; women: $\mathrm{PRR}=1.42[95 \% \mathrm{Cl}=1.14,1.76]$ ). The interaction term between employment status and sex was not significant $(p=0.71)$.
\end{abstract}

Conclusions: Temporary employment is adversely associated with oral health.

Keywords: Employment status, Number of teeth, Non-regular employment

\section{Background}

Oral diseases remain a significant public health problem due to their very high prevalence, major impact on quality of life [1], and costs on health care systems [2]. In addition, oral diseases are socially patterned and closely related to social deprivation [3]. Consequently, stark social inequalities in oral health are now a major public health concern [4].

Temporary employment has attracted the attention of health researchers in recent years, because it has significant adverse effects on health [5-9]. Owing to considerable changes in the labour markets, inferior working

\footnotetext{
* Correspondence: tsubo828@med.tohoku.ac.jp

'Department of International and Community Oral Health, Tohoku University Graduate School of Dentistry, 4-1, Seiryo-machi, Aoba-ku, Sendai, Miyagi, Japan

Full list of author information is available at the end of the article
}

conditions such as temporary contracts and an imbalanced working organization have emerged as a significant risk factor for poor health [10]. Unstable employment, such as temporary contracts, has been regarded as being harmful to health [5], and therefore, employment status might worsen health inequalities through employment status [5]. Temporary employment also may be harmful to oral health because work stress might lead to smoking tobacco [11] and decreasing salivary flow, which increases the risk of periodontal disease [12]. In addition, temporary employees might experience more severe tooth loss than regular ones, because their incomes are in general lower than ones of regular employees and they often do not receive adequate social benefits, such as health pensions [13]. 
A few studies have examined the relationship between employment status and oral health, including some that examined the association between unemployment and oral health [14-16]. To our knowledge, only one crosssectional study has reported significant associations between the workplace-related factors such as precarious employment status and poor self-rated oral health [17]. Our main hypothesis was that changes in employment status between regular and temporary employment would have a negative impact on tooth loss. The aim of this study was to examine whether the experience of temporary employment is associated with tooth loss among working adults in Japan.

\section{Methods}

\section{Data sources and participants}

We used data from the Japanese Study on Stratification, Health, Income, and Neighborhood (J-SHINE), which has been described in detail elsewhere [18]. This survey was conducted between July 2010 and February 2011. Target participants were adults aged $25-50$ years old from 4 municipalities in Japan (2 in the Tokyo metropolitan area and 2 in neighboring prefectures). Figure 1 shows a detailed flowchart of participant selection. A total of 13,920 participants were probabilistically selected from the residential registry. Trained survey staff successfully contacted 8408 community dwelling adults, and 4385 participants agreed to participate in the survey (response rate $31.5 \%$ ). The inclusion criteria were being $25-50$ years of age and being regular or temporary employees at initial (previous) and current employment. The exclusion criteria were having missing values among the independent or dependent variables and not having answered the survey questions by themselves. We excluded 68 participants who did not answer the survey questions by themselves, 1256 participants who did not answer the question about current employment status (regular and temporary), 43 participants who did not answer the question about initial employment status (regular and temporary), 52 participants who were not aged 25-50 years old, 4 participants who did not indicate their sex, and 310 participants who did not answer the question about tooth loss. The analytic population was 2652 participants (the details are shown in Fig. 1).

\section{Study design}

This study was a cross-sectional study.

\section{Independent variable: Changes in employment status}

We obtained information about current employment status from the question, "What is your employment? If you have several jobs, please answer about your main job." Respondents chose one answer from the following: "A president or an executive officer," "Regular employment," "Temporary employment," "Contract employment," "Part-time employment," "Self-employed," "Housekeeper," "Subsidiary jobs," and "Unknown." We categorized participants who chose the answer regular employment into the regular employment group and participants who chose the answers temporary

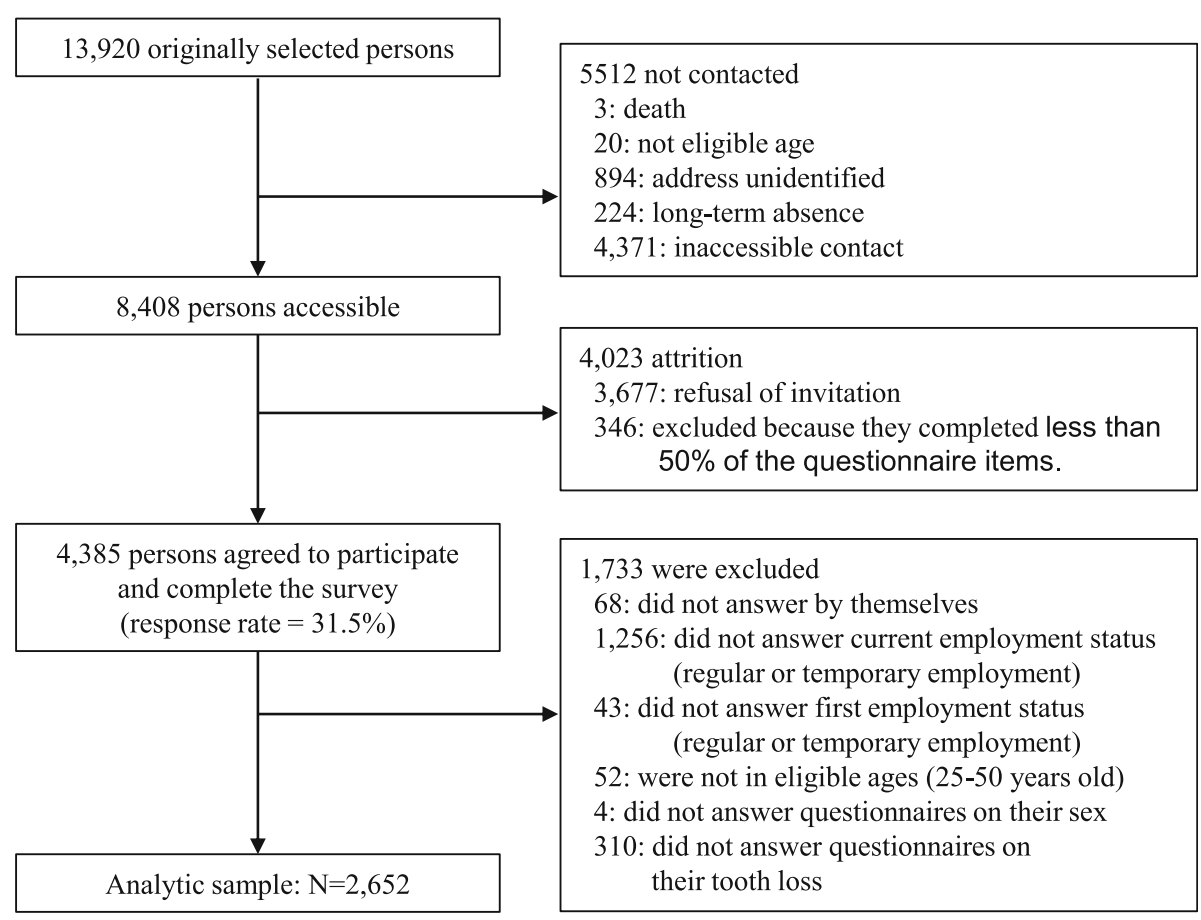

Fig. 1 A flowchart of participants in the present study 
employment, contract employment, or part-time employment as temporary employment. We excluded those who chose president or executive officer, self-employed, housekeeper, subsidiary jobs, or unknown in the categorization of initial or current employment status (see Fig. 1).

We asked all participants whether they had changed jobs. Among only those who had changed jobs, we obtained information about their previous (initial) employment status using the same questions posed for current employment status. For the main analysis, we used the replies about current and initial employment status to prepare two categories for the independent variable: continuous regular employment and the experience of temporary employment. For a more analysis, we created four categories: continuous regular employment (regular employee at both times), regular to temporary employment (regular employee at initial employment and temporary employee currently), temporary to regular employment (temporary employee at initial employment and regular employee currently), and continuous temporary employment (temporary employee at both times).

\section{Dependent variable: Self-reported tooth loss}

Dependent variable was self-reported tooth loss. We obtained this information using the question, "How many teeth have you had removed/extracted (excepting tooth extraction for orthodontic treatment, wisdom tooth extraction, and primary teeth)?" Respondents chose one of the following: "None" (scored 0$)$, "1 tooth" (scored 1), "2 teeth" (scored 2), "3 teeth" (scored 3), "4 teeth" (scored 4), and "more than 4 teeth" (scored 5). We used self-reported tooth loss as a count variable.

\section{Covariates}

We regarded the following factors as potential confounders, and included them in the multivariable adjusted models: age (categorized as $25-30,30-35,35-40$, $40-45$, or $45-50$ years) and sex (men or women). Health status variables that may be related to employment status and tooth loss were included: history of diabetes (none or present) and body mass index $\left(\mathrm{kg} / \mathrm{m}^{\wedge} 2\right)(\geq 25.0$, 18.5-25.0, or $<18.5)$. In addition, social determinants variables that could affect oral health were also included: years of education $(<9,10-12$, or $>12$ years $)$, self-rated household economic status in early life at 5 years old (rich, fair, or poor), marital status (married or single), and number of family members in the household (living alone, 2 , 3, or $\geq 4$ ).

We supposed potential pathways: income, psychological stress and disorders, access to health care, and health behavior. Annual household income $(0-300,300-750$, or $>750$ million Japanese yen) was also included. We used feeling fear of job loss (yes or no) and psychological distress (K6 score [19]; none (0-4) or present $(\geq 5))$ as a psychological stress and disorders variable. To assess the access to health care, we included visiting a dental clinic for preventative care (yes or no) and hesitation to use medical and dental care (yes, no, or never felt a need to use). We included smoking status (current smoker, former smoker, or never smoker) as a health behavior variable. We created dummy variables for the missing values for each covariate.

\section{Statistical analysis}

We conducted a negative binomial regression analysis stratified by sex to estimate prevalence rate ratios (PRRs) and $95 \%$ confidence intervals (95\%CIs) for tooth loss, because there are clear different trends of employment status between men and women in Japan [20, 21]. We also examined an interaction term between changes in employment status and sex adjusting for age. We created 2 models for adjusting potential confounders. In model 1, we controlled for age. In model 2 , years of education, self-rated household economic status in early life at 5 years old, marital status, and number of family members in the household, history of diabetes, and body mass index were added to model 1 . Subsequently, we constructed a model to evaluate how potential pathway variables explain the association. In model 3 , we added annual household income to model 2 . In model 4 , we added visiting a dental clinic for preventive care and hesitation to use medical and dental care to model 3. In model 5, we added feel fear of job loss and psychological distress to model 4. Finally, in model 6, we added smoking status to model 5 . We further conducted an analysis using 4 categories of independent variables to validate the findings of the main analysis. In addition, we conducted a linear regression analysis to confirm the validity of the results from a negative binomial regression analysis. We applied a chi-squared test for cross-tabulation. In addition, we constructed a directed acyclic graph (DAG) of proposed associations between employment status and tooth loss to guide our analyses (Fig. 2). $P$ values of $<0.05$ (two tailed) were considered significant. Analyses were conducted by using STATA ver. 14.2 (Stata Corp., College Station, TX).

\section{Results}

The median age was 37 years (first quartile to third quartile $=31$ to 43 ). More than half of the participants were men $(n=1394,52.6 \%)$. The percentage of the experience of temporary employment was $14.5 \%(n=202)$ in men and $61.3 \%(n=771)$ in women. Tables 1 and 2 show the characteristics and dependent variables among men and women. There was no significant association between men who experienced temporary employment and tooth loss. On the contrary, compared with women who were continuous regular employees, there was a significant association between women who experienced temporary employment and tooth loss. 


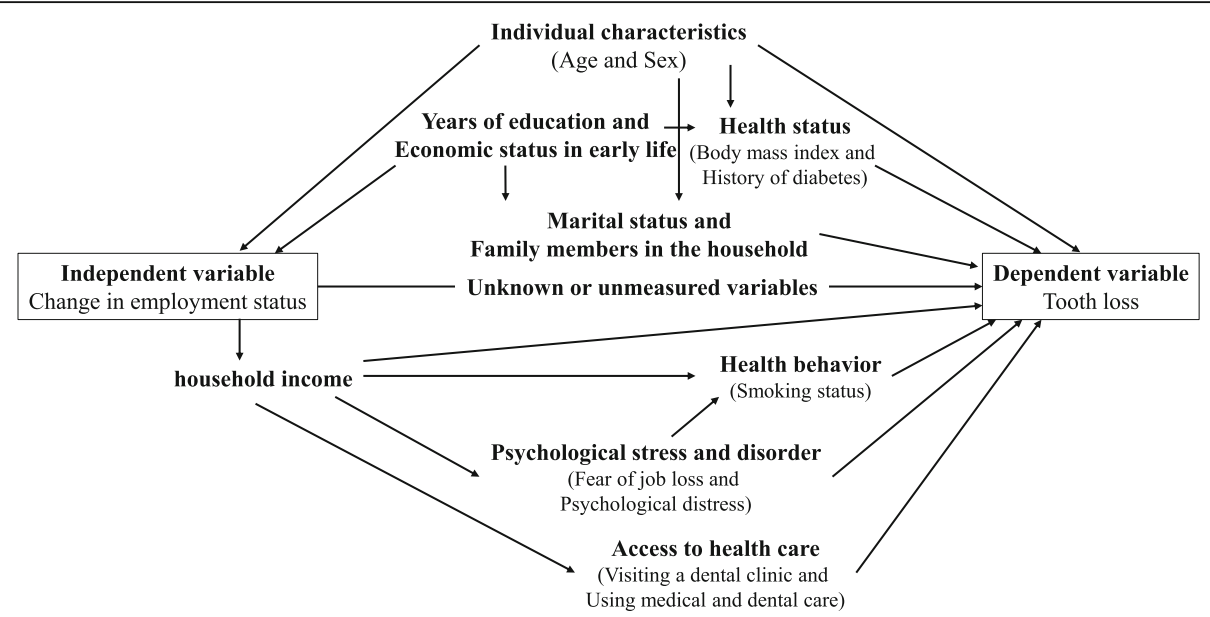

Fig. 2 A directed acyclic graph (DAG) showing the association between employment status and tooth loss

Table 3 shows the associations between change in employment status and tooth loss found with the multivariable ordered logistic regression models stratified by sex. We found no significant interaction between employment status and sex after adjusting for age $(p=0.71)$. In model 1, we confirmed a significant association between the experience of temporary employment and tooth loss in both sexes. Model 2 also showed that the experience of temporary employment was significantly associated with tooth loss after adjusting for potential confounders (men: PRR $=1.50[95 \% \mathrm{CI}=1.13,2.00]$; women: $\mathrm{PRR}=1.42$ $[95 \% \mathrm{CI}=1.14,1.76])$. In the additional analysis, compared with continuous regular employment, changes from regular to temporary employment and temporary to regular employment as well as continuous temporary employment were associated with tooth loss in models 1 and 2.

In models 3 to 6 , we observed associations between changes in employment status and tooth loss after adjusting for potential pathway variables. Compared with continuous regular employment, the PRR of having the experience of temporary employment decreased in models 3 to 6 (men, $\mathrm{PRR}=1.44[95 \% \mathrm{CI}=1.07,1.93]$ to 1.31 $[95 \% \mathrm{CI}=0.98,1.76]$; women, $\mathrm{PRR}=1.37[95 \% \mathrm{CI}=1.10$, $1.71]$ to $1.33[95 \% \mathrm{CI}=1.06,1.66])$. Similar trends were observed in the additional analysis of the regular to temporary employment, temporary to regular employment, and continuous temporary employment groups. The results from the linear regression analysis also showed similar trends with the main analysis.

\section{Discussions}

The results of our study showed that the experience of temporary employment was associated with tooth loss in both men and women in Japan. In addition, changes from regular to temporary employment and temporary to regular employment as well as continuous temporary employment were associated with tooth loss.

The association between temporary employment and poor oral health is important in public health because the level of unstable employment is increasing in both the private and public sectors in many developed countries [5]. The number of temporary employees continues to increase in these countries [9]: for example, the proportion of temporary employees in Japan was only $18.3 \%$ in 1988 but reached $37.4 \%$, or more than 1 in 3 workers, in 2014 [22]. Furthermore, more than half of employed young people (15-24 years old) in certain European countries are temporary workers: $53.6 \%$ in Germany, $57.1 \%$ in Italy, and 59.6\% in France in 2015 [23]. Dental health professionals and public policy makers should understand the enormous impact of increasing temporary employment on tooth loss.

We found that temporary employment was associated with tooth loss among both male and female workers in Japan. A previous survey of the labor force showed that the reasons for being temporarily employed differ between men and women. The primary reasons for temporary employment in men were "Can't find regular employment jobs" (26.9\%), whereas the reason in women was "work only during convenient time" (27.6\%) [24]. Therefore, it is conceivable that the association between temporary employment and oral health would also differ between sexes. That is, the negative effect of being temporarily employed would be amplified in men. However, the evidence suggests a different effect. Inoue et al. reported that temporary female employees faced precarious situations such as low income, limited social safety net, and difficulty sustaining work-life balance [21]. The current study also revealed that female participants who experienced temporary employment were low paid and fearful about job loss. Therefore, 
Table 1 Characteristics and tooth loss in men $(n=1,394)$

\begin{tabular}{|c|c|c|c|c|c|c|c|c|c|}
\hline \multicolumn{3}{|l|}{ Men $(n=1,394)$} & \multicolumn{6}{|c|}{ Number of tooth loss } & \multirow[b]{2}{*}{$P$-value* } \\
\hline & & & none & 1 tooth & 2 teeth & 3 teeth & 4 teeth & more than 4 teeth & \\
\hline \multirow[t]{10}{*}{ Changes in employment status } & $\begin{array}{l}\text { Continuous regular } \\
\text { employment }\end{array}$ & $\mathrm{n}$ & 736 & 140 & 111 & 60 & 46 & 99 & 0.68 \\
\hline & $(n=1,192)$ & $(\%)$ & $(61.7)$ & $(11.7)$ & (9.3) & $(5.0)$ & (3.9) & $(8.3)$ & \\
\hline & $\begin{array}{l}\text { Having the experience of } \\
\text { temporary employment }\end{array}$ & $\mathrm{n}$ & 122 & 20 & 23 & 9 & 6 & 22 & \\
\hline & $(n=202)$ & $(\%)$ & $(60.4)$ & (9.9) & (11.4) & $(4.5)$ & $(3.0)$ & $(10.9)$ & \\
\hline & $\begin{array}{l}\text { Regular to temporary } \\
\text { employment }\end{array}$ & $\mathrm{n}$ & 32 & 7 & 10 & 7 & 0 & 9 & \\
\hline & $(n=65)$ & $(\%)$ & $(49.2)$ & $(10.8)$ & $(15.4)$ & $(10.8)$ & $(0.0)$ & (13.9) & \\
\hline & $\begin{array}{l}\text { Temporary to regular } \\
\text { employment }\end{array}$ & $\mathrm{n}$ & 32 & 5 & 7 & 0 & 2 & 8 & \\
\hline & $(n=54)$ & $(\%)$ & $(59.3)$ & $(9.3)$ & (13.0) & $(0.0)$ & $(3.7)$ & $(14.8)$ & \\
\hline & $\begin{array}{l}\text { Continuous temporary } \\
\text { employment }\end{array}$ & $\mathrm{n}$ & 58 & 8 & 6 & 2 & 4 & 5 & \\
\hline & $(n=83)$ & $(\%)$ & $(69.9)$ & (9.6) & $(7.2)$ & $(2.4)$ & $(4.8)$ & $(6.0)$ & \\
\hline \multirow[t]{10}{*}{ Age (years old) } & $25-30$ & $\mathrm{n}$ & 220 & 18 & 15 & 3 & 4 & 6 & $<0.05$ \\
\hline & & $(\%)$ & $(82.7)$ & $(6.8)$ & $(5.6)$ & $(1.1)$ & $(1.5)$ & (2.3) & \\
\hline & $30-35$ & $\mathrm{n}$ & 169 & 22 & 15 & 11 & 7 & 16 & \\
\hline & & $(\%)$ & $(70.4)$ & $(9.2)$ & (6.3) & $(4.6)$ & $(2.9)$ & $(6.7)$ & \\
\hline & $35-40$ & $\mathrm{n}$ & 195 & 41 & 27 & 15 & 12 & 19 & \\
\hline & & $(\%)$ & $(63.1)$ & $(13.3)$ & (8.7) & $(4.9)$ & (3.9) & $(6.2)$ & \\
\hline & $40-45$ & $\mathrm{n}$ & 159 & 45 & 43 & 18 & 17 & 33 & \\
\hline & & $(\%)$ & $(50.5)$ & $(14.3)$ & (13.7) & $(5.7)$ & $(5.4)$ & (10.5) & \\
\hline & $45-50$ & $n$ & 115 & 34 & 34 & 22 & 12 & 47 & \\
\hline & & $(\%)$ & $(43.6)$ & $(12.9)$ & (12.9) & $(8.3)$ & $(4.6)$ & (17.8) & \\
\hline \multirow[t]{4}{*}{ History of diabetes } & None & $\mathrm{n}$ & 843 & 157 & 132 & 67 & 51 & 116 & 0.62 \\
\hline & & $(\%)$ & $(61.7)$ & $(11.5)$ & (9.7) & $(4.9)$ & $(3.7)$ & (8.5) & \\
\hline & Present & $n$ & 15 & 3 & 2 & 2 & 1 & 5 & \\
\hline & & $(\%)$ & (53.6) & $(10.7)$ & (7.1) & $(7.1)$ & (3.6) & $(17.9)$ & \\
\hline \multirow[t]{6}{*}{ Body mass index $(\mathrm{kg} / \mathrm{m} \wedge 2)$} & $\geq 25.0$ & $n$ & 214 & 53 & 31 & 20 & 14 & 40 & 0.11 \\
\hline & & $(\%)$ & $(57.5)$ & $(14.3)$ & (8.3) & $(5.4)$ & (3.8) & $(10.8)$ & \\
\hline & $18.5-25.0$ & $\mathrm{n}$ & 601 & 104 & 100 & 44 & 35 & 76 & \\
\hline & & $(\%)$ & (62.6) & $(10.8)$ & $(10.4)$ & $(4.6)$ & $(3.7)$ & $(7.9)$ & \\
\hline & $<18.5$ & $n$ & 38 & 3 & 2 & 5 & 3 & 3 & \\
\hline & & $(\%)$ & $(70.4)$ & $(5.6)$ & (3.7) & $(9.3)$ & $(5.6)$ & $(5.6)$ & \\
\hline \multirow[t]{4}{*}{ Marital status } & Married & $\mathrm{n}$ & 575 & 113 & 99 & 53 & 43 & 93 & $<0.05$ \\
\hline & & $(\%)$ & $(58.9)$ & $(11.6)$ & $(10.1)$ & $(5.4)$ & $(4.4)$ & (9.5) & \\
\hline & Single & $\mathrm{n}$ & 283 & 47 & 35 & 16 & 9 & 28 & \\
\hline & & $(\%)$ & $(67.7)$ & $(11.2)$ & (8.4) & (3.8) & $(2.2)$ & $(6.7)$ & \\
\hline \multirow{7}{*}{$\begin{array}{l}\text { No. of family members in } \\
\text { the household }\end{array}$} & Living alone & $n$ & 109 & 21 & 18 & 13 & 3 & 19 & 0.56 \\
\hline & & $(\%)$ & (59.6) & (11.5) & (9.8) & $(7.1)$ & (1.6) & (10.4) & \\
\hline & 2 & $n$ & 163 & 25 & 25 & 10 & 9 & 24 & \\
\hline & & (\%) & (63.7) & $(9.8)$ & (9.8) & (3.9) & (3.5) & (9.4) & \\
\hline & 3 & $n$ & 228 & 40 & 32 & 14 & 9 & 27 & \\
\hline & & $(\%)$ & $(65.1)$ & (11.4) & $(9.1)$ & $(4.0)$ & (2.6) & $(7.7)$ & \\
\hline & $\geq 4$ & $n$ & 357 & 74 & 59 & 32 & 31 & 51 & \\
\hline
\end{tabular}


Table 1 Characteristics and tooth loss in men ( $n=1,394)$ (Continued)

\begin{tabular}{|c|c|c|c|c|c|c|c|c|c|}
\hline \multicolumn{3}{|l|}{ Men $(n=1,394)$} & \multicolumn{6}{|c|}{ Number of tooth loss } & \multirow[b]{2}{*}{$P$-value* } \\
\hline & & & none & 1 tooth & 2 teeth & 3 teeth & 4 teeth & more than 4 teeth & \\
\hline & & (\%) & $(59.1)$ & $(12.3)$ & $(9.8)$ & (5.3) & $(5.1)$ & $(8.4)$ & \\
\hline \multirow{6}{*}{$\begin{array}{l}\text { Self-rated household economic status } \\
\text { in early life at } 5 \text { years old }\end{array}$} & Rich & $\mathrm{n}$ & 138 & 45 & 28 & 13 & 13 & 28 & 0.06 \\
\hline & & $(\%)$ & $(52.1)$ & $(17.0)$ & $(10.6)$ & $(4.9)$ & $(4.9)$ & $(10.6)$ & \\
\hline & Fair & $\mathrm{n}$ & 566 & 90 & 80 & 45 & 29 & 69 & \\
\hline & & (\%) & $(64.4)$ & $(10.2)$ & $(9.1)$ & $(5.1)$ & $(3.3)$ & $(7.9)$ & \\
\hline & Poor & $\mathrm{n}$ & 145 & 24 & 25 & 11 & 10 & 23 & \\
\hline & & (\%) & $(60.9)$ & $(10.1)$ & $(10.5)$ & $(4.6)$ & $(4.2)$ & $(9.7)$ & \\
\hline \multirow[t]{6}{*}{ Years of education (year) } & $<9$ & $\mathrm{n}$ & 31 & 3 & 6 & 6 & 1 & 6 & $<0.05$ \\
\hline & & (\%) & $(58.5)$ & (5.7) & $(11.3)$ & $(11.3)$ & $(1.9)$ & $(11.3)$ & \\
\hline & $9-12$ & $\mathrm{n}$ & 113 & 32 & 31 & 12 & 8 & 35 & \\
\hline & & (\%) & $(48.9)$ & $(13.9)$ & $(13.4)$ & $(5.2)$ & (3.5) & $(15.2)$ & \\
\hline & $>12$ & $\mathrm{n}$ & 708 & 124 & 96 & 51 & 42 & 80 & \\
\hline & & $(\%)$ & $(64.3)$ & (11.3) & $(8.7)$ & $(4.6)$ & (3.8) & $(7.3)$ & \\
\hline \multirow[t]{6}{*}{ Annual household income (million yen) } & $0-300$ & $\mathrm{n}$ & 35 & 10 & 7 & 5 & 1 & 9 & 0.86 \\
\hline & & $(\%)$ & $(52.2)$ & $(14.9)$ & $(10.5)$ & $(7.5)$ & $(1.5)$ & $(13.4)$ & \\
\hline & $300-750$ & $\mathrm{n}$ & 369 & 67 & 60 & 28 & 23 & 51 & \\
\hline & & (\%) & $(61.7)$ & $(11.2)$ & $(10.0)$ & $(4.7)$ & $(3.9)$ & $(8.5)$ & \\
\hline & $\geq 750$ & $\mathrm{n}$ & 287 & 59 & 47 & 26 & 16 & 46 & \\
\hline & & (\%) & $(59.7)$ & $(12.3)$ & $(9.8)$ & $(5.4)$ & $(3.3)$ & (9.6) & \\
\hline \multirow[t]{4}{*}{ Feel fear of job loss } & No & $\mathrm{n}$ & 563 & 103 & 81 & 43 & 25 & 68 & $<0.05$ \\
\hline & & $(\%)$ & (63.8) & $(11.7)$ & $(9.2)$ & $(4.9)$ & $(2.8)$ & $(7.7)$ & \\
\hline & Yes & $\mathrm{n}$ & 269 & 55 & 47 & 23 & 25 & 52 & \\
\hline & & $(\%)$ & $(57.1)$ & $(11.7)$ & $(10.0)$ & $(4.9)$ & $(5.3)$ & $(11.0)$ & \\
\hline \multirow[t]{4}{*}{ Psychological distress (k6) } & None (0-4) & $\mathrm{n}$ & 565 & 106 & 89 & 45 & 32 & 78 & 0.99 \\
\hline & & $(\%)$ & $(61.8)$ & (11.6) & $(9.7)$ & $(4.9)$ & $(3.5)$ & $(8.5)$ & \\
\hline & Present $(\geq 5)$ & $n$ & 293 & 54 & 44 & 24 & 20 & 42 & \\
\hline & & $(\%)$ & $(61.4)$ & $(11.3)$ & $(9.2)$ & $(5.0)$ & $(4.2)$ & $(8.8)$ & \\
\hline \multirow[t]{4}{*}{ Visiting a dental clinic for preventive care } & Yes & $\mathrm{n}$ & 201 & 38 & 24 & 25 & 11 & 27 & 0.12 \\
\hline & & (\%) & $(61.7)$ & $(11.7)$ & $(7.4)$ & $(7.7)$ & $(3.4)$ & $(8.3)$ & \\
\hline & No & $\mathrm{n}$ & 654 & 122 & 109 & 44 & 41 & 93 & \\
\hline & & (\%) & (61.5) & $(11.5)$ & $(10.3)$ & $(4.1)$ & (3.9) & $(8.8)$ & \\
\hline \multirow[t]{6}{*}{ Hesitation to use medical and dental care } & Yes & $\mathrm{n}$ & 374 & 78 & 58 & 28 & 28 & 69 & $<0.05$ \\
\hline & & $(\%)$ & $(58.9)$ & $(12.3)$ & $(9.1)$ & $(4.4)$ & $(4.4)$ & $(10.9)$ & \\
\hline & No & $\mathrm{n}$ & 353 & 70 & 55 & 34 & 18 & 38 & \\
\hline & & (\%) & $(62.2)$ & $(12.3)$ & $(9.7)$ & $(6.0)$ & $(3.2)$ & $(6.7)$ & \\
\hline & Never felt a need to use & $n$ & 131 & 12 & 21 & 6 & 6 & 14 & $<0.05$ \\
\hline & & (\%) & $(69.0)$ & (6.3) & $(11.1)$ & $(3.2)$ & $(3.2)$ & $(7.4)$ & \\
\hline \multirow[t]{6}{*}{ Smoking status } & Current smoker & $n$ & 258 & 61 & 62 & 29 & 26 & 58 & \\
\hline & & $(\%)$ & $(52.2)$ & $(12.4)$ & (12.6) & $(5.9)$ & $(5.3)$ & $(11.7)$ & \\
\hline & Former smoker & $n$ & 226 & 43 & 41 & 22 & 15 & 30 & \\
\hline & & (\%) & $(60.0)$ & (11.4) & $(10.9)$ & $(5.8)$ & $(4.0)$ & $(8.0)$ & \\
\hline & Never smoker & $n$ & 373 & 56 & 31 & 18 & 11 & 32 & \\
\hline & & (\%) & $(71.6)$ & $(10.8)$ & $(6.0)$ & (3.5) & $(2.1)$ & $(6.1)$ & \\
\hline
\end{tabular}


Table 2 Characteristics and tooth loss in women $(n=1,258)$

\begin{tabular}{|c|c|c|c|c|c|c|c|c|c|}
\hline \multirow[t]{2}{*}{ Women $(n=1,258)$} & & & \multicolumn{6}{|c|}{ Number of tooth loss } & \multirow[b]{2}{*}{$P$-value* } \\
\hline & & & none & 1 tooth & 2 teeth & 3 teeth & 4 teeth & more than 4 teeth & \\
\hline \multirow[t]{10}{*}{ Changes in employment status } & $\begin{array}{l}\text { Continuous regular } \\
\text { employment }\end{array}$ & $n$ & 349 & 63 & 29 & 15 & 12 & 19 & $<0.05$ \\
\hline & $(n=487)$ & $(\%)$ & $(71.7)$ & $(12.9)$ & (6.0) & (3.1) & $(2.5)$ & (3.9) & \\
\hline & $\begin{array}{l}\text { Having the experience of } \\
\text { temporary employment }\end{array}$ & $n$ & 449 & 116 & 66 & 47 & 26 & 67 & \\
\hline & $(n=771)$ & $(\%)$ & $(58.2)$ & $(15.1)$ & $(8.6)$ & (6.1) & $(3.4)$ & $(8.7)$ & \\
\hline & $\begin{array}{l}\text { Regular to temporary } \\
\text { employment }\end{array}$ & $n$ & 286 & 82 & 52 & 33 & 17 & 47 & \\
\hline & $(n=517)$ & $(\%)$ & (55.3) & $(15.9)$ & $(10.1)$ & (6.4) & (3.3) & $(9.1)$ & \\
\hline & $\begin{array}{l}\text { Temporary to regular } \\
\text { employment }\end{array}$ & $n$ & 39 & 7 & 2 & 2 & 0 & 5 & \\
\hline & $(n=55)$ & $(\%)$ & (70.9) & $(12.7)$ & (3.6) & (3.6) & $(0.0)$ & $(9.1)$ & \\
\hline & $\begin{array}{l}\text { Continuous temporary } \\
\text { employment }\end{array}$ & $\mathrm{n}$ & 124 & 27 & 12 & 12 & 9 & 15 & \\
\hline & $(n=199)$ & $(\%)$ & $(62.3)$ & (13.6) & (6.0) & $(6.0)$ & $(4.5)$ & $(7.5)$ & \\
\hline \multirow[t]{10}{*}{ Age (years old) } & $25-30$ & $n$ & 248 & 20 & 8 & 5 & 6 & 5 & $<0.05$ \\
\hline & & $(\%)$ & $(84.9)$ & (6.9) & $(2.7)$ & (1.7) & $(2.1)$ & $(1.7)$ & \\
\hline & $30-35$ & $n$ & 163 & 24 & 12 & 6 & 7 & 9 & \\
\hline & & (\%) & (73.8) & $(10.9)$ & (5.4) & (2.7) & $(3.2)$ & $(4.1)$ & \\
\hline & $35-40$ & $n$ & 152 & 47 & 21 & 8 & 4 & 16 & \\
\hline & & (\%) & $(61.3)$ & $(19.0)$ & $(8.5)$ & $(3.2)$ & (1.6) & $(6.5)$ & \\
\hline & $40-45$ & $n$ & 133 & 47 & 25 & 16 & 11 & 19 & \\
\hline & & $(\%)$ & $(53.0)$ & (18.7) & $(10.0)$ & (6.4) & $(4.4)$ & $(7.6)$ & \\
\hline & $45-50$ & $n$ & 102 & 41 & 29 & 27 & 10 & 37 & \\
\hline & & $(\%)$ & $(41.5)$ & $(16.7)$ & (11.8) & $(11.0)$ & $(4.1)$ & $(15.0)$ & \\
\hline \multirow[t]{4}{*}{ History of diabetes } & None & $n$ & 793 & 178 & 94 & 60 & 38 & 86 & 0.24 \\
\hline & & $(\%)$ & (63.5) & $(14.3)$ & (7.5) & $(4.8)$ & (3.0) & (6.9) & \\
\hline & Present & $n$ & 5 & 1 & 1 & 2 & 0 & 0 & \\
\hline & & $(\%)$ & $(55.6)$ & (11.1) & $(11.1)$ & (22.2) & $(0.0)$ & $(0.0)$ & \\
\hline \multirow[t]{6}{*}{ Body mass index $(\mathrm{kg} / \mathrm{m} \wedge 2)$} & $\geq 25.0$ & $n$ & 65 & 19 & 14 & 7 & 4 & 18 & $<0.05$ \\
\hline & & $(\%)$ & $(51.2)$ & $(15.0)$ & $(11.0)$ & (5.5) & $(3.2)$ & $(14.2)$ & \\
\hline & $18.5-25.0$ & $\mathrm{n}$ & 580 & 131 & 63 & 43 & 27 & 60 & \\
\hline & & $(\%)$ & $(64.2)$ & $(14.5)$ & $(7.0)$ & (4.8) & $(3.0)$ & $(6.6)$ & \\
\hline & $<18.5$ & $n$ & 113 & 25 & 13 & 11 & 7 & 7 & \\
\hline & & $(\%)$ & $(64.2)$ & $(14.2)$ & (7.4) & (6.3) & $(4.0)$ & $(4.0)$ & \\
\hline \multirow[t]{4}{*}{ Marital status } & Married & $n$ & 455 & 124 & 58 & 51 & 27 & 62 & $<0.05$ \\
\hline & & $(\%)$ & $(58.6)$ & $(16.0)$ & $(7.5)$ & (6.6) & (3.5) & $(8.0)$ & \\
\hline & Single & $n$ & 340 & 55 & 37 & 11 & 11 & 24 & \\
\hline & & $(\%)$ & $(71.1)$ & $(11.5)$ & (7.7) & (2.3) & $(2.3)$ & $(5.0)$ & \\
\hline \multirow{7}{*}{$\begin{array}{l}\text { No. of family members in } \\
\text { the household }\end{array}$} & Living alone & $n$ & 75 & 6 & 7 & 5 & 3 & 5 & 0.32 \\
\hline & & (\%) & $(74.3)$ & (5.9) & (6.9) & (5.0) & (3.0) & (5.0) & \\
\hline & 2 & $n$ & 161 & 43 & 15 & 10 & 6 & 21 & \\
\hline & & (\%) & (62.9) & (16.8) & $(5.9)$ & (3.9) & $(2.3)$ & $(8.2)$ & \\
\hline & 3 & $n$ & 201 & 42 & 34 & 15 & 12 & 22 & \\
\hline & & (\%) & $(61.7)$ & (12.9) & $(10.4)$ & $(4.6)$ & (3.7) & (6.8) & \\
\hline & $\geq 4$ & $n$ & 356 & 87 & 39 & 31 & 17 & 37 & \\
\hline
\end{tabular}


Table 2 Characteristics and tooth loss in women $(n=1,258)$ (Continued)

\begin{tabular}{|c|c|c|c|c|c|c|c|c|c|}
\hline \multirow[t]{2}{*}{ Women $(n=1,258)$} & & & \multicolumn{6}{|c|}{ Number of tooth loss } & \multirow[b]{2}{*}{$P$-value ${ }^{*}$} \\
\hline & & & none & 1 tooth & 2 teeth & 3 teeth & 4 teeth & more than 4 teeth & \\
\hline & & (\%) & $(62.8)$ & $(15.3)$ & (6.9) & $(5.5)$ & (3.0) & $(6.5)$ & \\
\hline \multirow{6}{*}{$\begin{array}{l}\text { Self-rated household economic status in } \\
\text { early life at } 5 \text { years old }\end{array}$} & Rich & $\mathrm{n}$ & 139 & 32 & 26 & 18 & 12 & 28 & $<0.05$ \\
\hline & & (\%) & $(54.5)$ & (12.6) & $(10.2)$ & $(7.1)$ & $(4.7)$ & $(11.0)$ & \\
\hline & Fair & $n$ & 490 & 111 & 57 & 33 & 16 & 42 & \\
\hline & & $(\%)$ & $(65.4)$ & $(14.8)$ & $(7.6)$ & $(4.4)$ & $(2.1)$ & $(5.6)$ & \\
\hline & Poor & $n$ & 162 & 35 & 12 & 11 & 10 & 15 & \\
\hline & & (\%) & $(66.1)$ & $(14.3)$ & $(4.9)$ & $(4.5)$ & $(4.1)$ & $(6.1)$ & \\
\hline \multirow[t]{6}{*}{ Years of education (year) } & $<9$ & $n$ & 17 & 6 & 1 & 6 & 2 & 3 & $<0.05$ \\
\hline & & (\%) & $(48.6)$ & $(17.1)$ & $(2.9)$ & $(17.1)$ & $(5.7)$ & (8.6) & \\
\hline & $9-12$ & $n$ & 127 & 37 & 25 & 15 & 11 & 25 & \\
\hline & & $(\%)$ & $(52.9)$ & $(15.4)$ & $(10.4)$ & $(6.3)$ & $(4.6)$ & $(10.4)$ & \\
\hline & $>12$ & $n$ & 647 & 135 & 68 & 41 & 25 & 57 & \\
\hline & & (\%) & $(66.5)$ & $(13.9)$ & $(7.0)$ & $(4.2)$ & $(2.6)$ & $(5.9)$ & \\
\hline \multirow[t]{6}{*}{ Annual household income (million yen) } & $0-300$ & $n$ & 53 & 9 & 5 & 3 & 3 & 12 & 0.41 \\
\hline & & (\%) & $(62.4)$ & (10.6) & $(5.9)$ & $(3.5)$ & $(3.5)$ & $(14.1)$ & \\
\hline & $300-750$ & $n$ & 249 & 58 & 39 & 21 & 9 & 28 & \\
\hline & & (\%) & (61.6) & $(14.4)$ & $(9.7)$ & $(5.2)$ & $(2.2)$ & $(6.9)$ & \\
\hline & $\geq 750$ & $n$ & 233 & 57 & 26 & 20 & 14 & 27 & \\
\hline & & (\%) & (61.8) & $(15.1)$ & $(6.9)$ & $(5.3)$ & $(3.7)$ & $(7.2)$ & \\
\hline \multirow[t]{4}{*}{ Feel fear of job loss } & No & $n$ & 495 & 123 & 56 & 38 & 23 & 45 & 0.09 \\
\hline & & $(\%)$ & $(63.5)$ & $(15.8)$ & $(7.2)$ & $(4.9)$ & $(3.0)$ & $(5.8)$ & \\
\hline & Yes & $n$ & 272 & 46 & 32 & 21 & 13 & 39 & \\
\hline & & $(\%)$ & $(64.3)$ & $(10.9)$ & (7.6) & $(5.0)$ & $(3.1)$ & $(9.2)$ & \\
\hline \multirow[t]{4}{*}{ Psychological distress (k6) } & None (0-4) & $n$ & 548 & 120 & 66 & 30 & 25 & 50 & $<0.05$ \\
\hline & & (\%) & $(65.3)$ & $(14.3)$ & $(7.9)$ & (3.6) & $(3.0)$ & $(6.0)$ & \\
\hline & Present $(\geq 5)$ & $n$ & 248 & 59 & 29 & 31 & 13 & 36 & \\
\hline & & $(\%)$ & $(59.6)$ & $(14.2)$ & $(7.0)$ & $(7.5)$ & $(3.1)$ & $(8.7)$ & \\
\hline \multirow[t]{4}{*}{ Visiting a dental clinic for preventive care } & Yes & $n$ & 247 & 57 & 40 & 27 & 18 & 20 & $<0.05$ \\
\hline & & (\%) & $(60.4)$ & (13.9) & $(9.8)$ & $(6.6)$ & $(4.4)$ & $(4.9)$ & \\
\hline & No & $n$ & 548 & 122 & 55 & 35 & 20 & 65 & \\
\hline & & (\%) & $(64.9)$ & $(14.4)$ & $(6.5)$ & $(4.1)$ & $(2.4)$ & $(7.7)$ & \\
\hline \multirow[t]{6}{*}{ Hesitation to use medical and dental care } & Yes & $\mathrm{n}$ & 366 & 80 & 43 & 34 & 13 & 39 & 0.45 \\
\hline & & $(\%)$ & (63.7) & $(13.9)$ & $(7.5)$ & $(5.9)$ & $(2.3)$ & $(6.8)$ & \\
\hline & No & $n$ & 310 & 77 & 36 & 23 & 17 & 39 & \\
\hline & & (\%) & $(61.8)$ & $(15.3)$ & $(7.2)$ & $(4.6)$ & $(3.4)$ & $(7.8)$ & \\
\hline & Never felt a need to use & $n$ & 122 & 22 & 16 & 5 & 8 & 8 & \\
\hline & & $(\%)$ & $(67.4)$ & $(12.2)$ & $(8.8)$ & $(2.8)$ & $(4.4)$ & $(4.4)$ & \\
\hline \multirow[t]{6}{*}{ Smoking status } & Current smoker & $n$ & 91 & 26 & 15 & 17 & 11 & 21 & $<0.05$ \\
\hline & & $(\%)$ & $(50.3)$ & $(14.4)$ & $(8.3)$ & $(9.4)$ & $(6.1)$ & $(11.6)$ & \\
\hline & Former smoker & $n$ & 115 & 34 & 20 & 12 & 5 & 20 & \\
\hline & & (\%) & $(55.8)$ & $(16.5)$ & $(9.7)$ & $(5.8)$ & $(2.4)$ & $(9.7)$ & \\
\hline & Never smoker & $n$ & 589 & 119 & 59 & 33 & 22 & 45 & \\
\hline & & (\%) & (67.9) & $(13.7)$ & (6.8) & (3.8) & (2.5) & $(5.2)$ & \\
\hline
\end{tabular}


Table 3 Associations between change in employment status and tooth loss

\begin{tabular}{|c|c|c|c|c|c|}
\hline & \multicolumn{5}{|c|}{ Changes in employment status } \\
\hline & $\begin{array}{l}\text { Continuous regular } \\
\text { employment }\end{array}$ & $\begin{array}{l}\text { Having the experience of } \\
\text { temporary employment }\end{array}$ & $\begin{array}{l}\text { Regular to temporary } \\
\text { employment }\end{array}$ & $\begin{array}{l}\text { Temporary to regular } \\
\text { employment }\end{array}$ & $\begin{array}{l}\text { Continuous temporary } \\
\text { employment }\end{array}$ \\
\hline $\begin{array}{l}\text { Negative binomial regression } \\
\text { models }\end{array}$ & Reference & PRR $(95 \% \mathrm{Cl})$ & PRR $(95 \% \mathrm{Cl})$ & PRR $(95 \% \mathrm{Cl})$ & PRR $(95 \% \mathrm{Cl})$ \\
\hline Men $(n=1,394)$ & $(n=1,192)$ & $(n=202)$ & $(n=65)$ & $(n=54)$ & $(n=83)$ \\
\hline Model 1 & 1.00 & $1.55(1.18,2.04)$ & $1.71(1.11,2.63)$ & $1.69(1.05,2.73)$ & $1.31(0.86,2.01)$ \\
\hline Model 2 & 1.00 & $1.50(1.13,2.00)$ & $1.62(1.05,2.52)$ & $1.62(0.99,2.64)$ & $1.30(0.83,2.02)$ \\
\hline Model 3 & 1.00 & $1.44(1.07,1.93)$ & $1.51(0.96,2.37)$ & $1.63(1.00,2.65)$ & $1.22(0.77,1.92)$ \\
\hline Model 4 & 1.00 & $1.38(1.03,1.85)$ & $1.44(0.91,2.26)$ & $1.53(0.94,2.50)$ & $1.20(0.76,1.88)$ \\
\hline Model 5 & 1.00 & $1.32(0.98,1.78)$ & $1.37(0.87,2.16)$ & $1.46(0.89,2.39)$ & $1.16(0.74,1.82)$ \\
\hline Model 6 & 1.00 & $1.31(0.98,1.76)$ & $1.41(0.90,2.21)$ & $1.43(0.88,2.33)$ & $1.13(0.72,1.77)$ \\
\hline Women $(n=1,258)$ & $(n=487)$ & $(n=771)$ & $(n=517)$ & $(n=55)$ & $(n=199)$ \\
\hline Model 1 & 1.00 & $1.44(1.16,1.79)$ & $1.34(1.06,1.70)$ & $1.33(0.79,2.24)$ & $1.73(1.28,2.34)$ \\
\hline Model 2 & 1.00 & $1.42(1.14,1.76)$ & $1.35(1.07,1.72)$ & $1.30(0.77,2.18)$ & $1.62(1.19,2.19)$ \\
\hline Model 3 & 1.00 & $1.37(1.10,1.71)$ & $1.31(1.02,1.66)$ & $1.31(0.78,2.20)$ & $1.56(1.14,2.12)$ \\
\hline Model 4 & 1.00 & $1.38(1.11,1.72)$ & $1.32(1.03,1.68)$ & $1.29(0.76,2.19)$ & $1.58(1.16,2.15)$ \\
\hline Model 5 & 1.00 & $1.37(1.09,1.71)$ & $1.32(1.03,1.70)$ & $1.27(0.75,2.17)$ & $1.51(1.10,2.06)$ \\
\hline Model 6 & 1.00 & $1.33(1.06,1.66)$ & $1.31(1.02,1.68)$ & $1.14(0.67,1.94)$ & $1.44(1.06,1.97)$ \\
\hline Linear regression models & Reference & Coefficient $(95 \% \mathrm{Cl})$ & Coefficient $(95 \% \mathrm{Cl})$ & Coefficient $(95 \% \mathrm{Cl})$ & Coefficient $(95 \% \mathrm{Cl})$ \\
\hline Men $(n=1,394)$ & $(n=1,192)$ & $(n=202)$ & $(n=65)$ & $(n=54)$ & $(n=83)$ \\
\hline Model 1 & - & $0.38(0.14,0.62)$ & $0.51(0.12,0.91)$ & $0.47(0.04,0.90)$ & $0.21(-0.15,0.57)$ \\
\hline Model 2 & - & $0.37(0.12,0.62)$ & $0.46(0.06,0.85)$ & $0.42(-0.01,0.85)$ & $0.25(-0.12,0.62)$ \\
\hline Model 3 & - & $0.34(0.09,0.59)$ & $0.41(0.01,0.81)$ & $0.42(-0.02,0.85)$ & $0.22(-0.16,0.59)$ \\
\hline Model 4 & - & $0.32(0.07,0.57)$ & $0.38(-0.02,0.78)$ & $0.38(-0.05,0.81)$ & $0.23(-0.15,0.60)$ \\
\hline Model 5 & - & $0.28(0.02,0.53)$ & $0.33(-0.07,0.74)$ & $0.31(-0.12,0.74)$ & $0.20(-0.18,0.58)$ \\
\hline Model 6 & - & $0.25(0.00,0.50)$ & $0.31(-0.09,0.71)$ & $0.29(-0.15,0.72)$ & $0.17(-0.20,0.55)$ \\
\hline Women $(n=1,258)$ & $(n=487)$ & $(n=771)$ & $(n=517)$ & $(n=55)$ & $(n=199)$ \\
\hline Model 1 & - & $0.25(0.08,0.41)$ & $0.19(0.00,0.38)$ & $0.25(-0.16,0.65)$ & $0.36(0.13,0.60)$ \\
\hline Model 2 & - & $0.23(0.06,0.40)$ & $0.20(0.00,0.39)$ & $0.25(-0.15,0.65)$ & $0.31(0.07,0.54)$ \\
\hline Model 3 & - & $0.20(0.03,0.38)$ & $0.17(-0.03,0.36)$ & $0.24(-0.16,0.64)$ & $0.27(0.02,0.51)$ \\
\hline Model 4 & - & $0.21(0.04,0.39)$ & $0.17(-0.02,0.37)$ & $0.24(-0.16,0.65)$ & $0.28(0.04,0.53)$ \\
\hline Model 5 & - & $0.20(0.02,0.38)$ & $0.16(-0.04,0.36)$ & $0.25(-0.15,0.65)$ & $0.26(0.01,0.51)$ \\
\hline Model 6 & - & $0.16(-0.02,0.33)$ & $0.13(-0.06,0.33)$ & $0.11(-0.29,0.51)$ & $0.22(-0.03,0.46)$ \\
\hline
\end{tabular}

Model 1: Age was adjusted

Model 2: Model 1 + years of education, self-rated household economic status in early life at 5 years old, marital status, no. of family members in the household,

history of diabetes, and body mass index were adjusted

Model 3: Model $2+$ Annual household income was adjusted

Model 4: Model 3 + Visiting a dental clinic for preventive care and hesitation to use medical and dental care were adjusted

Model 5: Model $4+$ Feel fear of job loss and psychological distress was adjusted

Model 6: Model $5+$ Smoking status was adjusted

Abbreviation: PRR prevalence rate ratios, $95 \% \mathrm{Cl} 95 \%$ confidence interval

temporary employment could affect tooth loss in both sexes uniformly.

Several potential pathways can exist between temporary employment and oral health. First, economic factors may link employment status and oral health. In general, temporary employees have incomes lower than those of regular employees, and low income is among the key risk factors for oral disease [25]. Low income is associated with severe caries and periodontal disease, and poor people are less likely to use medical services [26]. Indeed, the association between temporary employment and tooth loss was explained by the analysis of income in the present study (models 2 and 3 ). 
Second, psychological stress and disorders may explain the association between temporary employment and tooth loss. Because they can be easily dismissed, temporary employees tend to feel more job insecurity and work-related stress which lead to psychological disorders $[7,13,27]$. Stress from fear of job loss and psychological disorders could influence health behaviors such as less frequent toothbrushing and heavier smoking [11]. In addition, stress may decrease salivary flow, which increases the occurrence and progression of periodontal disease [12]. Temporary employees could lose their teeth for any of these reasons. Indeed, the association between temporary employment and tooth loss was explained by the fear of job loss and psychological disorders in the present analysis (models 4 and 5).

Third, poor health behavior also might explain the association between employment status and oral health. Work stress was associated with poor health behaviors such as less frequent toothbrushing and heavy smoking [11]. In addition, low social economic status could lead to poor oral health behaviors [26]. Indeed, the association between temporary employment and tooth loss was explained by smoking status (models 5 and 6). However, we could not obtain data on oral health behavior variables such as toothbrushing. It might also well explain the association between temporary employment and tooth loss.

Finally, limited access to health care might explain the association between employment status and oral health. Japan has universal healthcare coverage (UHC) and patients pay only $10-30 \%$ of the total cost of treatment [28]. Also, the total cost itself is relatively low because the cost is controlled by the government. In addition, the UHC covers the most basic dental treatments, such as treatments for caries and periodontal disease [28]. With the UHC, most people in Japan did not hesitate obtaining medical and dental services. However, under long lasting economic depression, some people in temporary employment, a new emerging type of unstable employment, were not able to use health care service appropriately due to the following two reasons [29]; 1) even $10-30 \%$ of the total cost of dental care could be a barrier for them to use dental care because they were employed at a low wage, 2) they may be reluctant to take a time off from work to visit dental services because they are concerned that they might be fired if they are absent frequently owing to sickness. Indeed, the association between temporary employment and tooth loss was explained by the frequency of visiting a dental clinic for preventive care and the hesitation to use medical and dental care as analyzed in our study (models 3 and 4).

The present study has limitations. First, both the independent and dependent variables were self-reported, which may have introduced self-reporting bias. Although, several studies have shown that the validity and reliability of self-reported oral health status are acceptable [30], selfrated number of teeth lost is not validated. However, previous studies have used self-reported number of teeth lost $[31,32]$. Second, the response rate was relatively low, which could be another source of bias. However, the respondents had characteristics that were fairly comparable to those of the target population [18]. Therefore, our findings are likely to be generalizable in Japan.

\section{Conclusions}

In conclusion, we found a significant association between temporary employment and tooth loss. A previous study indicated that there is a need to enhance the social safety net for temporary employees even in high-income countries [5]. Secure employment is a social determinant of health [5], and the assurance of safety/physical protections in workplaces, health insurance, and more stable employment arrangements are needed. Policy makers as well as dental health professionals should understand the impact of employment status on population health.

\section{Abbreviations \\ 95\%Cl: 95\% confidence interval; J-SHINE: Japanese Study on Stratification, Health, Income, and Neighborhood; PRR: Prevalence rate ratios; UHC: universal healthcare coverage; WHO: World Health Organization}

\section{Acknowledgements}

The authors would like to thank participants and the team for providing us with data from the Japanese Study of Stratification, Health, Income, and Neighborhood (J-SHINE).

\section{Funding}

This research was supported by a Grand-in-Aid for Scientific Research on Innovative Areas (No. 21119002) and a Grant-in-Aid for Scientific Research (B) (No. 15H04781) from the Ministry of Education, Culture, Sports, Science and Technology, Japan.

\section{Availability of data and materials}

The data that support the findings of this study are available with the JSHINE research team, but restrictions apply to the availability of these data, which were used with permission for the current study; therefore, data are not publicly available. However, it can be available upon reasonable request and with permission from the J-SHINE research team.

\section{Authors' contributions}

YS: conception and design, analysis and interpretation of data, and drafting the article. TT: acquisition of data, analysis and interpretation of data, and drafting the article. RW, JA, and KO: analysis and interpretation of data. All authors revised it critically and approved the final manuscript.

\section{Ethics approval and consent to participate}

The J-SHINE study's ethics approval and informed consent procedure were reviewed and approved by the ethics committee of the Graduate School of Medicine and Faculty of Medicine at the University of Tokyo. Informed consent was obtained in writing from all participants. We obtained permission from the J-SHINE research team to access and use the data for our study.

Consent for publication

Not applicable.

Competing interests

The authors declare that they have no competing interests. 


\section{Publisher's Note}

Springer Nature remains neutral with regard to jurisdictional claims in published maps and institutional affiliations.

\section{Author details}

'Department of International and Community Oral Health, Tohoku University Graduate School of Dentistry, 4-1, Seiryo-machi, Aoba-ku, Sendai, Miyagi, Japan. ${ }^{2}$ Department of Epidemiology and Public Health, University College London, Gower Street, London WC1E 6BT, UK.

Received: 18 July 2017 Accepted: 14 February 2018 Published online: 21 February 2018

\section{References}

1. Marcenes W, Kassebaum NJ, Bernabé E, Flaxman A, Naghavi M, Lopez A et al. Global burden of oral conditions in 1990-2010: a systematic analysis. J Dent Res. 2013;92:592-7.

2. Listl S, Galloway J, Mossey PA, Marcenes W. Global economic impact of dental diseases. J Dent Res. 2015;94:1355-61.

3. Watt RG, Sheiham A. Integrating the common risk factor approach into a social determinants framework. Community Dent Oral Epidemiol. 2012:40:289-96.

4. Sheiham A, Alexander D, Cohen L, Marinho V, Moysés S, Petersen PE, et al. Global oral health inequalities: task group-implementation and delivery of oral health strategies. Adv Dent Res. 2011;23:259-67.

5. Benach J, Muntaner C, Santana V. Employment conditions knowledge network (EMCONET). Employ. Cond. Heal. inequalities. Final Rep. to WHO Comm. Soc. Determ. Heal. 2007.

6. Virtanen $M$, Kivimäki $M$, Joensuu $M$, Virtanen $P$, Elovainio $M$, Vahtera J. Temporary employment and health: a review. Int J Epidemiol. 2005; 34:610-22.

7. Virtanen $\mathrm{P}$, Janlert $\mathrm{U}$, Hammarström A. Exposure to temporary employment and job insecurity: a longitudinal study of the health effects. Occup Environ Med. 2011;68:570-4.

8. Inoue A, Kawakami N, Tsuchiya M, Sakurai K, Hashimoto H. Association of occupation, employment contract, and company size with mental health in a national representative sample of employees in Japan. J Occup Health. 2010;52:227-40.

9. Kivimäki M, Vahtera J, Virtanen $\mathrm{M}$, Elovainio M, Pentti J, Ferrie JE. Temporary employment and risk of overall and cause-specific mortality. Am J Epidemiol. 2003;158:663-8.

10. Berkman LF, Kawachi I, Theorell T. Working Conditions and Health. Soc. Epidemiol. New York: Oxford University Press; 2014. p. 153-81.

11. Wardle J, Steptoe A, Oliver G, Lipsey Z. Stress, dietary restraint and food intake. J Psychosom Res. 2000;48:195-202.

12. Marcenes WS, Sheiham A. The relationship between work stress and oral health status. Soc Sci Med. 1992;35:1511-20.

13. Ishiguro K. Japanese employment in transformation: the growing number of non-regular workers. Electron. J. Contemp. Japanese Stud. . 2008;

14. Al-Sudani FYH, Vehkalahti MM, Suominen AL. The association between current unemployment and clinically determined poor oral health. Community Dent Oral Epidemiol. 2015:43:325-37.

15. Al-Sudani FYH, Vehkalahti MM, Suominen AL. Association of current employment status with oral health-related behaviors: findings from the Finnish health 2000 survey. Eur J Oral Sci. 2016;124:368-76.

16. Mundt T, Schwahn C, Mack F, Polzer I, Samietz S, Kocher T, et al. Risk indicators for missing teeth in working-age Pomeranians-an evaluation of high-risk populations. J Public Health Dent. 2007;67:243-9.

17. Tsuboya T, Aida J, Kawachi I, Katase K, Osaka K. Early life-course socioeconomic position, adult work-related factors and oral health disparities: cross-sectional analysis of the J-SHINE study. BMJ Open. 2014;4:e005701.

18. Takada M, Kondo N, Hashimoto H. J-SHINE data management committee. Japanese study on stratification, health, income, and neighborhood: study protocol and profiles of participants. J. Epidemiology. 2014;24:334-44.

19. Kessler RC, Andrews G, Colpe L, Hiripi E, Mroczek DK, Normand SLT, et al. Short screening scales to monitor population prevalences and trends in non-specific psychological distress. Psychol Med. 2002;32:959-76.

20. Estévez-abe M. An international comparison of gender Equality : why is the Japanese gender gap so Persistent? Japan Labor Rev. 2013;10:82-100.
21. Inoue M, Nishikitani M, Tsurugano S. Female non-regular workers in Japan: their current status and health. Ind Health. 2016:54:521-7.

22. The Japan Institute for Labour Policy and Training. Labor Situation in Japan and Its Analysis: General Overview 2015/2016. p. 2016.

23. Organisation for Economic Co-operation and Development. OECD Employment Outlook 2016. Paris: OECD Publishing; 2016.

24. Annual report on the labour force survey. Minist. Tokyo: Intern. Aff. Commun; 2015

25. Steele J, Shen J, Tsakos G, Fuller E, Morris S, Watt R, et al. The interplay between socioeconomic inequalities and clinical oral health. J Dent Res. 2015;94:19-26.

26. Watt RG. Social determinants of oral health inequalities: implications for action. Community Dent Oral Epidemiol. 2012;40:44-8.

27. De Witte H. Job insecurity: review of the international literature on definitions, prevalence, antecedents and consequences. SA J Ind Psychol. 2005:31:1-6.

28. Ikegami N, Yoo B-K, Hashimoto $H$, Matsumoto M, Ogata H, Babazono A, et al. Japanese universal health coverage: evolution, achievements, and challenges. Lancet (London, England). Elsevier Ltd. 2011;378:1106-15.

29. Inoue M, Nishikitani M, Tsurugano S, Yano E. The health of permanent workers and workers with precarious employment: a literature review. Sangyo eiseigaku zasshi. 2011:53:117-39.

30. Matsui D, Yamamoto T, Nishigaki M, Miyatani F, Watanabe I, Koyama T, et al. Validity of self-reported number of teeth and oral health variables. BMC Oral Health BMC Oral Health. 2017;17:17.

31. Haugejorden O, Klock KS, Trovik TA. Incidence and predictors of selfreported tooth loss in a representative sample of Norwegian adults. Community Dent Oral Epidemiol. 2003;31:261-8.

32. Hung H-C, Joshipura KJ, Colditz G, Manson JE, Rimm EB, Speizer FE, et al. The association between tooth loss and coronary heart disease in men and women. J Public Health Dent. 2004:64:209-15.

\section{Submit your next manuscript to BioMed Central and we will help you at every step:}

- We accept pre-submission inquiries

- Our selector tool helps you to find the most relevant journal

- We provide round the clock customer support

- Convenient online submission

- Thorough peer review

- Inclusion in PubMed and all major indexing services

- Maximum visibility for your research

Submit your manuscript at www.biomedcentral.com/submit
Biomed Central 